\title{
BMJ Open Clinician, patient and general public beliefs about diagnostic imaging for low back pain: protocol for a qualitative evidence synthesis
}

\author{
Adrian C Traeger, ${ }^{1}$ Benjamin J Reed, ${ }^{2,3}$ Denise A O'Connor, ${ }^{2,3}$ Tammy C Hoffmann, ${ }^{4}$ \\ Gustavo C Machado, ${ }^{1}$ Carissa Bonner, ${ }^{1}$ Chris G Maher, ${ }^{1,5}$ Rachelle Buchbinder ${ }^{2,3}$
}

To cite: Traeger AC, Reed BJ, O'Connor DA, et al. Clinician, patient and general public beliefs about diagnostic imaging for low back pain: protocol for a qualitative evidence synthesis. BMJ Open 2018;8:e019470. doi:10.1136/ bmjopen-2017-019470

- Prepublication history and additional material for this paper are available online. To view these files, please visit the journal online (http://dx.doi. org/10.1136/bmjopen-2017019470).

Received 6 September 2017 Revised 20 December 2017 Accepted 2 January 2018

Check for updates

${ }^{1}$ School of Public Health, University of Sydney, Sydney, New South Wales, Australia ${ }^{2}$ Monash Department of Clinical Epidemiology, Cabrini Institute, Malvern, Victoria, Australia ${ }^{3}$ Department of Epidemiology and Preventive Medicine, School of Public Health and Preventive Medicine, Monash University, Melbourne, Victoria, Australia ${ }^{4}$ Faculty of Health Sciences and Medicine, Centre for Research in Evidence-Based Practice, Bond University, Gold Coast, Queensland, Australia

${ }^{5}$ Institute for Musculoskeletal Health, Sydney Local Health District, Sydney, New South Wales, Australia

Correspondence to

Dr Adrian C Traeger;

adrian.traeger@sydney.edu.au

\section{ABSTRACT}

Introduction Little is known about how to reduce unnecessary imaging for low back pain. Understanding clinician, patient and general public beliefs about imaging is critical to developing strategies to reduce overuse. Objective To synthesise qualitative research that has explored clinician, patient or general public beliefs about diagnostic imaging for low back pain.

Methods and analysis We will perform a qualitative evidence synthesis of relevant qualitative research exploring clinician, patient and general public beliefs about diagnostic imaging for low back pain. Exclusions will be studies not using qualitative methods and studies not published in English. Studies will be identified using sensitive search strategies in MEDLINE, EMBASE, CINAHL, AMED and PsycINFO. Two reviewers will independently apply inclusion and exclusion criteria, extract data, and use the Critical Appraisal Skills Programme quality assessment tool to assess the quality of included studies. To synthesise the data we will use a narrative synthesis approach that involves developing a theoretical model, conducting a preliminary synthesis, exploring relations in the data, and providing a structured summary. We will code the data using NVivo. At least two reviewers will independently apply the thematic framework to extracted data. Confidence in synthesis findings will be evaluated using the GRADE Confidence in the Evidence from Reviews of Qualitative Research tool.

Ethics and dissemination Ethical approval is not required to conduct this review. We will publish the results in a peer-reviewed journal.

PROSPERO registration number CRD42017076047.

\section{INTRODUCTION}

Low back pain is the leading cause of disability worldwide, and is responsible for an estimated 83 million years lived with disability per year. ${ }^{1}$ In 2013, healthcare-related costs of low back pain were estimated to be \$A5 billion in Australia ${ }^{2}$ and US\$87.6 billion (\$A110.5 billion) in the USA. ${ }^{3}$ Much of this cost is related to unnecessary tests and treatments. For example, diagnostic imaging is an unnecessary test for the vast majority of
Strengths and limitations of this study

- We will use systematic search strategies to identify relevant qualitative research.

- A priori methods will reduce bias related to searching, data extraction, study quality assessment and rating of confidence in synthesis findings.

- At least two authors will independently develop and refine a thematic framework to code data within included studies.

- Synthesis is limited to quotes and themes explored in the original reports.

patients with low back pain who present to primary care, yet in 2014, Australians spent \$A214 million on lumbar radiographs. ${ }^{4}$ Internationally, rates of guideline-discordant diagnostic imaging across primary, secondary and emergency department settings have been reported to be as high as $55 \% .^{5-12}$

Unnecessary diagnostic imaging is associated with substantial harm including the risk of overdiagnosis. ${ }^{13}$ Overdiagnosis occurs when diagnostic imaging detects incidental findings that are common in the asymptomatic population (eg, intervertebral disc degeneration ${ }^{14}$ ) and provides the patient with a diagnostic label that brings them no benefit or causes harm. Diagnostic labelling leads to medical overuse, a problem which is growing internationally. ${ }^{15}$ Unnecessary diagnostic imaging for low back pain drives flow-on effects such as overuse of advanced imaging, opioid prescriptions, spinal injections and surgery ${ }^{16}{ }^{17}$ Evidence from clinical guidelines suggests that most of these interventions have little to no benefit, and substantial risk for harms, in patients with non-specific low back pain. ${ }^{18-20}$

Little is known about how to reduce unnecessary imaging. A recent systematic review found that of the five trials located on 
strategies to reduce imaging in primary care, only one was successful. ${ }^{21}$ That trial found evidence that distribution of radiological guidelines plus audit and feedback on practice imaging rates reduced spinal imaging by $20 \%(95 \%$ CI 3\%-37\%) compared with control. ${ }^{22}$ We are aware of one other successful trial in primary care. In 1986, Deyo and colleagues tested an approach that combined patient education with a delayed referral for imaging. ${ }^{23}$ Though it was not the primary outcome for the trial, the authors found the delayed approach substantially reduced imaging rates (control imaging rate $83.9 \%$ vs intervention imaging rate $27.8 \%) .{ }^{23}$ These results have yet to be replicated.

There is robust evidence that a delayed approach reduces unnecessary antibiotic prescriptions for acute respiratory infections. ${ }^{24}$ Such an approach is promising for low back pain because, like many acute respiratory infections, natural recovery often occurs in the first 2 weeks. ${ }^{25}$ A delayed referral for imaging would see many patients improve before they made the final decision to pursue imaging or not. Clinical guidelines endorse a delayed approach for most patients with low back pain with the exception of suspected cauda equina syndrome or vertebral infection. ${ }^{18}$ Survey data from the trial by Deyo et al suggested that delaying imaging was acceptable to patients. ${ }^{23}$ However, such data provide little insight into the key, underlying beliefs about diagnostic imaging that could be gained, for example, from a qualitative study design.

Understanding clinician, patient and general public beliefs is an important step towards developing an acceptable delayed imaging approach. Although some clinician-level barriers to providing appropriate imaging for low back pain have been identified, ${ }^{26}$ it is not known whether the same barriers would impact on a decision to delay a request for imaging. For example, while clinicians tend to believe patients expect imaging, ${ }^{26}$ it is unclear how clinicians might perceive the benefits and harms of delaying, or avoiding, a referral. A previous qualitative synthesis found patients with chronic musculoskeletal pain value a diagnosis, but did not explore what participants believed about the role of diagnostic imaging. ${ }^{27}$ Similarly, surveys suggest around $50 \%$ of patients and the general public expect diagnostic imaging to manage low back pain, ${ }^{28-30}$ but it is unclear what people believe diagnostic imaging can actually provide; such beliefs could determine the acceptability of a delayed approach. For example, patients and the general public may be unwilling to accept a delay if they believe it will preclude effective diagnosis and treatment. ${ }^{31}$

We are not aware of any up-to-date qualitative evidence syntheses that could inform the use of the delayed imaging strategy for low back pain.

\section{AIM}

To synthesise qualitative research that has explored clinician, patient or general public beliefs about diagnostic imaging for low back pain.

\section{METHODS}

We have registered this study on PROSPERO (CRD42017076047).

\section{Selection criteria}

We will include English language articles that fulfil the following criteria:

Types of studies

Used qualitative methods for data collection (eg, focus groups, interviews) and analysis. All qualitative designs will be included and studies that included mixed methods (qualitative and quantitative), where the qualitative data were collected and analysed independently to the quantitative data, will also be included.

\section{Types of participants}

Clinicians who treat low back pain (eg, general practitioner, rheumatologist, spine surgeon, physiotherapist, chiropractor, osteopath, and so on), patients with low back pain of any duration, or the general public interviewed about management of low back pain. Participants do not necessarily need to have received diagnostic imaging.

\section{Types of settings}

Any healthcare (primary, secondary or tertiary care) or non-healthcare setting in any country.

\section{Types of imaging}

Any diagnostic imaging (eg, X-ray, CT scan, MRI, and so on).

Types of outcome measures (findings on phenomena of interest) Analysis describes beliefs about diagnostic imaging for low back pain. Beliefs are defined as 'a proposition or a set of propositions held true. ${ }^{32}$ We are interested in all beliefs about diagnostic imaging, not just those related to delaying or avoiding the test.

\section{Search methods}

We will develop a search strategy with the assistance of an Information Specialist based at the University of Sydney Library, and by using guidance from the Cochrane Qualitative and Implementation Methods Group (http:// methods.cochrane.org/qi/supplemental-handbookguidance). To locate relevant articles, we will search the following electronic databases: MEDLINE, EMBASE, CINAHL, AMED and PsycINFO. We will not use date limits, but will limit the search to articles in English. Sampling will be comprehensive rather than purposive; comprehensive searching will allow us to locate all relevant studies representing the phenomenon of interest (ie, beliefs about imaging for low back pain). We will perform the search in two parts as suggested by DeJean et $a l^{33}$ Part 1 aims to identify all studies on our topic of interest. The search strategy for part 1 will combine terms recommended by the Cochrane Back and Neck Review Group to identify studies of low back pain, terms describing the participants of interest (clinicians, patients, general public) and terms describing the phenomenon of interest (beliefs). Because the term 'beliefs' can be used interchangeably with others such as 'views', we will 
Table 1 Template for organising full-text studies into themes

\begin{tabular}{lll} 
& Theme 1 & Theme 2 \\
\hline $\begin{array}{l}\text { Study } \\
\text { (date) }\end{array}$ & $\begin{array}{l}\text { Supports theme/does not } \\
\text { support theme/no data }\end{array}$ & \\
\hline
\end{tabular}

develop a list of terms that are conceptually related to beliefs. For example, we will include broad terms such as 'perceptions,' 'feelings,' 'views' and 'understandings' (online supplementary appendix). Part 2 will aim to identify all studies using qualitative methodology. Where available we will use qualitative filters to maximise sensitivity and specificity of searches to locate qualitative studies in part 2, and adapt these to the other electronic databases (EMBASE, CINAHL, PsycINFO). ${ }^{33-35}$ To obtain final search results we will combine parts 1 and 2 using the syntax 'AND'. An example search strategy for MEDLINE is provided in the online supplementary appendix. We will also search the reference lists and conduct citation tracking of included studies using Web of Science. To identify other potentially relevant studies we will contact experts in the field through existing professional networks of the review authors.

\section{Selection of studies}

One reviewer will download search results to reference manager software and delete duplicates. To detect duplicates we will use the Systematic Review Assistant-Deduplication Module which has been shown to improve efficiency of the deduplication process. ${ }^{36}$ Two reviewers will screen titles and abstracts independently and perform full-text review as necessary. Disagreements about which studies to include will be resolved through discussion. If consensus cannot be reached an additional reviewer will make the final decision. The search and screening results will be presented in the form of a flow diagram as recommended by the Preferred Reporting Items for Systematic Reviews and Meta-Analyses checklist. ${ }^{37}$

\section{Assessment of quality of included studies}

There is currently no consensus on standard criteria to be used to assess the methodological quality of qualitative studies. For this qualitative evidence synthesis we will use the Critical Appraisal Skills Programme (CASP) quality assessment tool for qualitative studies. ${ }^{38}$ Two reviewers will independently apply the CASP tool to the included studies. The tool evaluates rigour, credibility and relevance of qualitative research. Disagreements will be resolved by consensus or by discussion with an additional reviewer. Studies will not be excluded on the basis of quality but quality assessments will be used to determine confidence in the synthesis findings.

\section{Data extraction}

Two reviewers will use a standard form to extract descriptive data from included studies (study details, design, sample details, and so on) and independently perform data extraction from $50 \%$ of the included studies. A third reviewer will extract descriptive data from all included studies and cross-check. Disagreements will be resolved by consensus or by discussion with a fourth reviewer. The descriptive data to extract will include:

- Study details (authors, year of publication).

- Research question.

- Design (phenomenological, thematic analysis, grounded theory, case study, ethnography, mixed methods).

- Participants (demographic characteristics, number, study location, history of low back pain and/or care seeking for low back pain, professional involved or professional background (eg, general practitioner, rheumatologist, spine surgeon, physiotherapist, chiropractor, osteopath, and so on)).

- Setting (country; urban/rural; primary, secondary, tertiary care).

- Diagnostic imaging type (eg, X-ray, CT, MRI, other, not specified).

- Method(s) (eg, focus group, interview) and timing in relation to seeking care (for patients with low back pain) of data collection and analysis, including if any theory or framework was used.

- Study findings. These will include themes, subthemes, discussion points and conclusions about imaging beliefs based on the original study authors' interpretation of the first order constructs (ie, 'second order constructs'). ${ }^{39}$ This will be supported by specific beliefs expressed by study participants in the primary study in the form of quotes (ie, 'first order constructs').$^{39}$ To extract data on study findings, two reviewers will constantly update their own extraction sheets to include new headings (ie, themes) as they emerge. This process forms part of the thematic analysis described in more detail in the section below.

\section{Data analysis and synthesis}

We plan to synthesise findings using a narrative approach. ${ }^{40}$ We chose a narrative synthesis method described by Popay et $a l^{40}$ because of its suitability to the mixed methods expertise of our review team, the type of data we are seeking (information from a large number of studies, rather than a small number of conceptually rich studies), the potential relevance of aggregative synthesis results to policymakers, clinicians and commissioners of research, the comprehensive sampling technique, and the realist epistemological position. ${ }^{41}$ The synthesis will involve four steps conducted in an iterative process rather than sequentially ${ }^{40}$ :

a. Developing a theoretical model.

b. Developing a preliminary synthesis.

c. Exploring relationships in the data and emerging themes.

d. Providing a structured summary and assessing the robustness of review findings. 
Table 2 Template for summary of qualitative findings

\begin{tabular}{|c|c|c|c|c|c|c|c|}
\hline $\begin{array}{l}\text { Review } \\
\text { finding }\end{array}$ & $\begin{array}{l}\text { Studies } \\
\text { contributing to } \\
\text { review finding }\end{array}$ & $\begin{array}{l}\text { Assessment of } \\
\text { methodological } \\
\text { limitations }\end{array}$ & $\begin{array}{l}\text { Assessment } \\
\text { of relevance }\end{array}$ & $\begin{array}{l}\text { Assessment } \\
\text { of coherence }\end{array}$ & $\begin{array}{l}\text { Assessment } \\
\text { of adequacy }\end{array}$ & $\begin{array}{l}\text { Overall GRADE } \\
\text { CERQual } \\
\text { assessment of } \\
\text { confidence }\end{array}$ & $\begin{array}{l}\text { Explanation } \\
\text { of } \\
\text { judgement }\end{array}$ \\
\hline Finding 1 & & & & & & & \\
\hline
\end{tabular}

CERQual, Confidence in the Evidence from Reviews of Qualitative Research.

\section{Developing a theoretical model}

We plan to develop our theoretical model iteratively while performing open coding of the data, using constant comparison between existing themes and new data to incorporate new themes as they emerge. We will consider applying specific theories of behaviour change to contextualise our synthesis findings at the final stage of analysis, if they reflect the data adequately. However, we will not constrain the initial coding and synthesis procedures with an overarching existing theory. ${ }^{40}$

\section{Developing a preliminary synthesis}

We will perform a preliminary synthesis of the findings by reading a diverse subset of included studies in detail and identifying the themes that emerge. To choose the subset of studies we will use purposive sampling based on key study features likely to influence beliefs, such as participants involved (clinicians, patients, general public), timing of interviews in relation to care seeking, healthcare setting, and duration of symptoms.

Two review authors will code findings from the same subset of studies and develop a preliminary list of themes. The review team will discuss the preliminary list of themes and revise the list in response to new themes that emerge in the remaining studies. We will tabulate this final list of themes and use it to organise findings from all studies. We will use NVivo software to facilitate coding processes. The final list of themes and accompanying data will be agreed on by discussion and consensus among the review team.

We will tabulate the full-text papers according to the presence or absence of key themes, with supporting data

Box 1 GRADE Confidence in the Evidence from Reviews of Qualitative Research (CERQual) approach ${ }^{43}$ to confidence in findings of qualitative evidence synthesis

High confidence: highly likely that the review finding is a reasonable representation of the phenomenon of interest.

Moderate confidence: likely that the review finding is a reasonable representation of the phenomenon of interest.

Low confidence: possible that the review finding is a reasonable representation of the phenomenon of interest.

Very low confidence: unclear whether the review finding is a reasonable representation of the phenomenon of interest. where available (eg, text extracts and participant quotes; table 1). We acknowledge that a theme occurring in more than one study does not provide evidence that the theme is important or common. However, we consider the number studies supporting a theme to contribute to the certainty that the theme is relevant to the phenomenon we are interested in.

\section{Exploring relationships in the data}

We will use two methods to explore relationships in the data: examining consistency of themes and idea webbing. ${ }^{42}$ In this step the 'data' will be second order constructs-themes, subthemes, discussion points, conclusions and selected participant quotes about imaging beliefs based on the original study authors' interpretation of views expressed by participants. To examine consistency of themes identified in the preliminary analysis we will explore data extracted from studies with different characteristics such as healthcare setting (eg, primary, secondary, tertiary care), timing of interviews and duration of pain (ie, acute: $<3$ months, chronic: 3 months or longer). To further explore relationships in the data we will use idea webbing. Idea webbing maps major themes and subthemes using web diagrams to identify cross-cutting, contradictory or overlapping themes. ${ }^{42}$ Two reviewers will independently develop their own idea webs and decide on a final diagram after discussion with the review team.

\section{Providing a structured summary}

We will provide a structured summary of synthesis findings in the form of a table (table 2). The table will include a summary of the robustness of the synthesis findings according to the GRADE Confidence in the Evidence from Reviews of Qualitative Research (CERQual) approach. ${ }^{43}$ The GRADE CERQual approach is described further below.

\section{Assessment of confidence in review findings}

We will use the CERQual tool to assess confidence in our findings. ${ }^{43}$ This tool considers four factors that influence the confidence in syntheses of qualitative studies: (1) methodological limitations of the studies contributing to the review finding; (2) the relevance of contributing studies to the review finding; (3) the coherence of data contributing to the review finding; and (4) the adequacy of data contributing to the review finding.

Methods on how to operationalise the CERQual items are still in development. ${ }^{43}$ Below we describe how we 
plan to operationalise these items for this review. Criteria will be used as a guide only; at least two independent reviewers will make a subjective judgement about confidence in each review finding. Reviewers will judge overall confidence in a review finding as high, moderate, low, or very low, with a justification for this rating (box 1). Each finding will begin with a rating of high confidence; reviewers will downgrade if there are concerns with any of the four CERQual criteria. A final decision on confidence in review findings will be reached through discussion and consensus among the review team. To assist with the GRADE CERQual approach we will use draft worksheets that the GRADE CERQual Working Group have provided to us, on request, via http://www.cerqual. org.

Methodological limitations of the studies contributing to the review finding

To examine item 1 we will use the CASP tool to consider the quality of studies contributing to the finding. If many of the contributing studies have important problems with design or conduct we will consider that to raise 'major concerns' about methodological limitations.

\section{Relevance of contributing studies to review finding}

To examine item 2 we will consider factors such as healthcare setting, duration of low back pain (acute or chronic), history of care seeking, timing of interviews (before or after consultation in care seekers), professional background (for clinician participants), socioeconomic background, and reimbursement system for imaging (publicly subsidised or free, privately funded). If studies contributing to review finding are based on data that have contextual features that do not relate to our review question, or to our objective to develop strategies to reduce overuse of imaging, we will consider that to raise major concerns about relevance.

\section{Coherence of data contributing to review finding}

To examine item 3 we will create a table to depict the number of studies that contributed to a given finding. Rows will contain primary studies, columns will contain higher order themes and subthemes (table 1). If some of the data supporting a review finding are ambiguous, or contradict the review finding, we will consider that to raise major concerns about coherence.

\section{Adequacy of data contributing to review finding}

To examine item 4 we will consider the quantity and 'richness' of the data supporting a review finding. We consider rich descriptions to be those which include detailed narrative descriptions, such as quotes that allow the reader to visualise the participant-researcher interaction and provide sufficient detail to support or refute the phenomenon of interest. If most studies lack rich narrative descriptions, we will consider that to raise major concerns about adequacy of data.

\section{Reporting}

We will report this study in accordance with the Enhancing Transparency in Reporting the synthesis of Qualitative research (ENTREQ) statement. ${ }^{44}$

\section{DISCUSSION}

Here we present the design of a qualitative evidence synthesis to explore beliefs about diagnostic imaging for low back pain. We anticipate this review will provide a robust picture on how healthcare professionals, patients and society might view the utility of spinal imaging. Understanding such beliefs is critical to determining the feasibility of strategies aimed at reducing unnecessary use, including those that encourage a delayed referral.

Contributors ACT, BR, CGM and RB conceived the idea for the study. ACT wrote the first draft of the manuscript. BR, DAO, TH, GCM, CB, CGM and RB contributed to revising subsequent drafts critically for important intellectual content. ACT, BR, DAO, $\mathrm{TH}, \mathrm{GCM}, \mathrm{CB}, \mathrm{CGM}$ and RB approved the final version of the manuscript and agree to be accountable for its content.

Funding This work was supported by an Australian National Health and Medical Research Council (NHMRC) programme grant entitled 'Using healthcare wisely: reducing inappropriate use of tests and treatments' (APP1113532). ACT, GCM and $\mathrm{CB}$ are supported by NHMRC Early Career Fellowships. CM is supported by an NHMRC Principal Research Fellowship. RB is supported by an NHMRC Senior Principal Research Fellowship.

Competing interests None declared.

Patient consent Not required.

Provenance and peer review Not commissioned; externally peer reviewed.

Open Access This is an Open Access article distributed in accordance with the Creative Commons Attribution Non Commercial (CC BY-NC 4.0) license, which permits others to distribute, remix, adapt, build upon this work non-commercially, and license their derivative works on different terms, provided the original work is properly cited and the use is non-commercial. See: http://creativecommons.org/ licenses/by-nc/4.0/

(c) Article author(s) (or their employer(s) unless otherwise stated in the text of the article) 2018. All rights reserved. No commercial use is permitted unless otherwise expressly granted.

\section{REFERENCES}

1. Vos T, Barber RM, Bell B, et al. Global, regional, and national incidence, prevalence, and years lived with disability for 301 acute and chronic diseases and injuries in 188 countries, 1990-2013: a systematic analysis for the Global Burden of Disease Study 2013. Lancet 2015;386:743-800.

2. Access Economics. A problem worth solving: the rising cost of musculoskeletal conditions. A report. Elsternwick, Victoria, 2013.

3. Dieleman JL, Baral R, Birger M, et al. US spending on personal health care and public health, 1996-2013. JAMA 2016;316:2627-46.

4. Australian Government Department of Health. MBS online: commonwealth of Australia. 2017 http://www9.health.gov.au/mbs/ search.cfm?q=spine\&sopt=S (accessed 14 Jul 2017).

5. Gil S, Quilis N, Vela P. FRI0557 Assessment of the Appropriateness of Lumbar Spine Magnetic Resonance Imaging in a Rheumatology Unit. Ann Rheum Dis 2015;74:629.2-629.

6. Kovacs FM, Arana E, Royuela A, et al. Appropriateness of lumbar spine magnetic resonance imaging in Spain. Eur J Radiol 2013;82:1008-14.

7. Espinoza CPT, Skupin M, Montezuma D, et al. Adherence to guidelines for low back pain imaging: comparison of a teaching and non-teaching clinic. J Gen Intern Med 2012;27:S113-S14.

8. Isaacs DM, Marinac J, Sun C. Radiograph use in low back pain: a United States Emergency Department database analysis. J Emerg Med 2004;26:37-45. 
9. González-Urzelai V, Palacio-Elua L, López-de-Munain J. Routine primary care management of acute low back pain: adherence to clinical guidelines. Eur Spine J 2003;12:589-94.

10. Gold R, Esterberg E, Hollombe C, et al. Low back imaging when not indicated: a descriptive cross-system analysis. Perm J 2016;20:25-33.

11. Jame SZ, Sari AA, Majdzadeh R, et al. The extent of inappropriate use of magnetic resonance imaging in low back pain and its contributory factors. Int J Prev Med 2014;5:1029-36.

12. Gidwani $R$, Sinnott $P$, Avoundjian $T$, et al. Inappropriate ordering of lumbar spine magnetic resonance imaging: are providers Choosing Wisely? Am J Manag Care 2016;22:e68-76.

13. Flynn TW, Smith B, Chou R. Appropriate use of diagnostic imaging in low back pain: a reminder that unnecessary imaging may do as much harm as good. J Orthop Sports Phys Ther 2011:41:838-46.

14. Brinjikji W, Luetmer PH, Comstock B, et al. Systematic literature review of imaging features of spinal degeneration in asymptomatic populations. AJNR Am J Neuroradiol 2015;36:811-6.

15. Brownlee S, Chalkidou K, Doust J, et al. Evidence for overuse of medical services around the world. Lancet 2017;390:156-68.

16. Webster BS, Bauer AZ, Choi Y, et al. latrogenic consequences of early magnetic resonance imaging in acute, work-related, disabling low back pain. Spine 2013;38:1939-46.

17. Webster BS, Choi Y, Bauer AZ, et al. The cascade of medical services and associated longitudinal costs due to nonadherent magnetic resonance imaging for low back pain. Spine 2014;39:1433-40.

18. Chou R, Qaseem A, Owens DK, et al. Diagnostic imaging for low back pain: advice for high-value health care from the American College of Physicians. Ann Intern Med 2011;154:181-9.

19. Qaseem A, Wilt TJ, McLean RM, et al. Noninvasive Treatments for Acute, Subacute, and Chronic Low Back Pain: A Clinical Practice Guideline From the American College of Physicians. Ann Intern Med 2017:166:514-30.

20 National Institute for Health and Care Excellence (NICE): Clinical Guidelines. Low Back Pain and Sciatica in Over 16s: assessment and management. 2016.

21. Jenkins HJ, Hancock MJ, French SD, et al. Effectiveness of interventions designed to reduce the use of imaging for low-back pain: a systematic review. CMAJ 2015;187:401-8

22. Kerry $S$, Oakeshott $P$, Dundas $D$, et al. Influence of postal distribution of the Royal College of Radiologists' guidelines, together with feedback on radiological referral rates, on X-ray referrals from general practice: a randomized controlled trial. Fam Pract 2000;17:46-52.

23. Deyo RA, Diehl AK, Rosenthal M. Reducing roentgenography use. Can patient expectations be altered? Arch Intern Med 1987;147:141-5

24. Spurling GK, Del Mar CB, Dooley L, et al. Delayed antibiotics for respiratory infections. Cochrane Database Syst Rev 2013:CD004417.

25. da C Menezes Costa L, Maher CG, Hancock MJ, et al. The prognosis of acute and persistent low-back pain: a meta-analysis. CMAJ 2012;184:E613-E624.

26. Slade SC, Kent P, Patel S, et al. Barriers to Primary Care Clinician Adherence to Clinical Guidelines for the Management of Low Back Pain: A Systematic Review and Metasynthesis of Qualitative Studies. Clin J Pain 2016;32:800-16.
27. Toye F, Seers K, Allcock N, et al. Patients' experiences of chronic non-malignant musculoskeletal pain: a qualitative systematic review. Br J Gen Pract 2013;63:829-41.

28. Carey M, Turon H, Goergen S, et al. Patients' experiences of the management of lower back pain in general practice: use of diagnostic imaging, medication and provision of self-management advice. Aust J Prim Health 2015;21:342-6.

29. Klaber Moffett JA, Newbronner E, Waddell G, et al. Public perceptions about low back pain and its management: a gap between expectations and reality? Health Expect 2000;3:161-8.

30. Ihlebaek C, Eriksen HR. Myths and perceptions of back pain in the Norwegian population, before and after the introduction of guidelines for acute back pain. Scand J Public Health 2005;33:401-6.

31. Verbeek J, Sengers MJ, Riemens L, et al. Patient expectations of treatment for back pain: a systematic review of qualitative and quantitative studies. Spine 2004;29:2309-18.

32. VandenBos GR. APA dictionary of psychology: American Psychological Association, 2007.

33. DeJean D, Giacomini M, Simeonov D, et al. Finding Qualitative Research Evidence for Health Technology Assessment. Qual Health Res 2016;26:1307-17.

34. Wilczynski NL, Marks S, Haynes RB. Search strategies for identifying qualitative studies in CINAHL. Qual Health Res 2007;17:705-10.

35. Booth A. Searching for qualitative research for inclusion in systematic reviews: a structured methodological review. Syst Rev 2016;5:74.

36. Rathbone J, Carter M, Hoffmann T, et al. Better duplicate detection for systematic reviewers: evaluation of Systematic Review AssistantDeduplication Module. Syst Rev 2015;4:6.

37. Liberati A, Altman DG, Tetzlaff J, et al. The PRISMA statement for reporting systematic reviews and meta-analyses of studies that evaluate healthcare interventions: explanation and elaboration. BMJ 2009;339:b2700.

38. Milton Keynes Primary Care Trust. CASP Qualititative Checklist - 10 questions to help you make sense of qualitative research. Critical Skills Appraisal Programme http://www.casp-uk.net/checklists (accessed 8 August 2017)

39. Schütz A. Collected Papers 1. The Hague: Martinus Nijhoff, 1962.

40. Popay J, Roberts H, Sowden A, et al. Guidance on the conduct of narrative synthesis in systematic reviews. A product from the ESRC methods programme Version. 2006;1:b92.

41. Booth A, Noyes J, Flemming K, et al. Guidance on choosing qualitative evidence synthesis methods for use in health technology assessments of complex interventions. $2016 \mathrm{http}: / / \mathrm{www}$.integratehta.eu/downloads/ (accessed 6 Nov 2017).

42. Arai L, Britten N, Popay J, et al. Testing methodological developments in the conduct of narrative synthesis: a demonstration review of research on the implementation of smoke alarm interventions. Evidence \& Policy 2007;3:361-83.

43. Lewin S, Glenton C, Munthe-Kaas H, et al. Using qualitative evidence in decision making for health and social interventions: an approach to assess confidence in findings from qualitative evidence syntheses (GRADE-CERQual). PLoS Med 2015;12:e1001895.

44. Tong A, Flemming K, Mclnnes E, et al. Enhancing transparency in reporting the synthesis of qualitative research: ENTREQ. BMC Med Res Methodol 2012;12:181. 